\title{
Development, Optimization, and Evaluation of Carvedilol-Loaded Solid Lipid Nanoparticles for Intranasal Drug Delivery
}

\author{
Heba M. Aboud, ${ }^{1}$ Mohammed H. El komy, ${ }^{1}$ Adel A. Ali, ${ }^{1,3}$ Shahira F. El Menshawe, ${ }^{1}$ and Ahmed Abd Elbary ${ }^{2}$
}

Received 20 August 2015; accepted 19 October 2015; published online 7 January 2016

\begin{abstract}
Carvedilol, a beta-adrenergic blocker, suffers from poor systemic availability (25\%) due to firstpass metabolism. The aim of this work was to improve carvedilol bioavailability through developing carvedilol-loaded solid lipid nanoparticles (SLNs) for nasal administration. SLNs were prepared by emulsion/solvent evaporation method. A $2^{3}$ factorial design was employed with lipid type (Compritol or Precirol), surfactant (1 or $2 \% w / v$ poloxamer 188$)$, and co-surfactant $(0.25$ or $0.5 \% w / v$ lecithin $)$ concentrations as independent variables, while entrapment efficiency (EE\%), particle size, and amount of carvedilol permeated/unit area in $24 \mathrm{~h}\left(Q_{24}\right)$ were the dependent variables. Regression analysis was performed to identify the optimum formulation conditions. The in vivo behavior was evaluated in rabbits comparing the bioavailability of carvedilol after intravenous, nasal, and oral administration. The results revealed high drug EE\% ranging from 68 to $87.62 \%$. Carvedilol-loaded SLNs showed a spherical shape with an enriched core drug loading pattern having a particle size in the range of 66 to $352 \mathrm{~nm}$. The developed SLNs exhibited significant high amounts of carvedilol permeated through the nasal mucosa as confirmed by confocal laser scanning microscopy. The in vivo pharmacokinetic study revealed that the absolute bioavailability of the optimized intranasal SLNs (50.63\%) was significantly higher than oral carvedilol formulation $(24.11 \%)$. Hence, we conclude that our developed SLNs represent a promising carrier for the nasal delivery of carvedilol.
\end{abstract}

KEY WORDS: bioavailability; carvedilol; factorial design; intranasal; solid lipid nanoparticles.

\section{INTRODUCTION}

Carvedilol is a nonselective beta-adrenergic antagonist used in the treatment of hypertension and angina pectoris. Although it is well absorbed in the gastrointestinal tract, it is extensively metabolized in the liver leading to decreased bioavailability of about $25 \%$ (1). Accordingly, increasing carvedilol bioavailability is warranted for enhancing its therapeutic effectiveness.

Solid lipid nanoparticles (SLNs) represent an alternative carrier to traditional colloidal carriers such as liposomes, emulsions, and biodegradable polymeric nanoparticles. SLNs are prepared by replacing the liquid lipid (oil) of an $\mathrm{o} / \mathrm{W}$ emulsion with a solid lipid or a mixture of solid lipids (2). SLNs have numerous advantages that include (1) low-cost ingredients, ease of preparation, and scale up; (2) rapid dispersion in aqueous media and high entrapment of hydrophobic drugs with controlled particle size; (3) high

\footnotetext{
${ }^{1}$ Department of Pharmaceutics, Faculty of Pharmacy, Beni-Suef University, Beni-Suef, Egypt.

${ }^{2}$ Department of Pharmaceutics, Faculty of Pharmacy, Cairo University, Cairo, Egypt.

${ }^{3}$ To whom correspondence should be addressed. (e-mail: adel.ali@pharm.bsu.edu.eg)
}

biodegradability and biocompatibility with improved storage stabilities; and (4) ability to enhance bioavailability and targeting of several drugs (3). Therefore, we thought it will be beneficial to formulate carvedilol in SLNs.

It seems intuitive and tempting to prepare carvedilolloaded SLNs for oral delivery. However, peroral delivery of drug-loaded SLNs is hindered by burst release in gastric media (4). To overcome this drawback, intraduodenal administration $(5,6)$ and protective coating of SLNs have been prescribed (7). The former approach is clinically inadequate for repeated administration drugs, and the latter increases the costs of manufacture because of the additional coating step. An alternative approach to address the artifacts of SLNs oral administration is to evaluate other routes.

Unlike oral delivery, intranasal delivery is not associated with hepatic first-pass effect, inactivation by intestinal enzymes, or degradation through gastric acid. Additionally, the high vasculature of the nasal membranes facilitates drug absorption (8). Thus, we decided to investigate the utility of the nasal route for delivering carvedilol-loaded SLNs. This decision was further supported by the fact that several small molecules were successfully delivered by the nasal route through formulation of SLNs (9-11).

The aim of the present study was to improve carvedilol bioavailability through developing carvedilol-loaded SLNs for nasal administration. 


\section{MATERIALS AND METHODS}

\section{Materials}

Carvedilol was obtained as a gift sample from Global Napi Pharmaceutical Company (Egypt); glyceryl behenate (Compritol 888 ATO) and glyceryl palmitostearate (Precirol ATO 5) were kindly donated by Gattefossé (France); L- $\alpha$ phosphatidylcholine (soybean lecithin), poloxamer 407, poloxamer 188, polyacrylic acid (Carbopol 971P), formic acid (HPLC grade), acetonitrile (HPLC grade), disodium hydrogen phosphate, and dialysis bags with a molecular weight cutoff of 12,000 g/mol were purchased from Sigma-Aldrich (USA); and rhodamine B was purchased from Loba Chemie (India). All other ingredients used were of analytical grade.

\section{Experimental Design}

A $2^{3}$ full factorial design was used to investigate the effect of three independent variables: lipid type $\left(X_{1}\right)$, surfactant concentration $\left(X_{2}\right)$, and co-surfactant concentration $\left(X_{3}\right)$ on three dependent variables: entrapment efficiency $\left(Y_{1}\right)$, particle size $\left(Y_{2}\right)$, and amount of carvedilol permeated/unit area in $24 \mathrm{~h} Q_{24}\left(Y_{3}\right)$. The details of the design are shown in Table I.

\section{Preparation of Carvedilol-Loaded SLNs}

SLNs were prepared according to a modified emulsion/ solvent evaporation method (12). Briefly, $10 \mathrm{ml}$ organic phase was prepared by dissolving $10 \mathrm{mg}$ carvedilol, $100 \mathrm{mg}$ lipid (Compritol or Precirol), and lecithin (in concentrations of 0.25 and $0.5 \% w / v)$ in methylene chloride, while the aqueous phase was $25 \mathrm{ml}$ solution of poloxamer 188 in concentrations of 1 or $2 \% w / v$ in distilled water. Both phases were heated to the same temperature $\left(70^{\circ} \mathrm{C}\right.$ in the case of Compritol and $60^{\circ} \mathrm{C}$ in the case of Precirol). The organic phase was added dropwise to the hot aqueous phase at a constant rate under homogenization (Ultra Turrax ${ }^{\circledR}$ T 25 basic homogenizer, IKA, Staufen, Germany) and sonication (Sonix TV ss-series ultrasonicator, Sonix IV Ultrasonic Cleaning Systems, North Charleston, SC). Homogenization was carried out at $13,500 \mathrm{rpm}$ for $10 \mathrm{~min}$. The resulting colloidal dispersion was poured into $25 \mathrm{ml}$ cold distilled water and stirred at $1000 \mathrm{rpm}$ for $1 \mathrm{~h}$ at $2^{\circ} \mathrm{C}$ in an ice bath to allow for the hardening of the SLNs.

This nanoemulsion was centrifuged (SIGMA 3-30K, Steinheim, Germany) at $14,000 \mathrm{rpm}$ for $3 \mathrm{~h}$ at $4^{\circ} \mathrm{C}$. The precipitate was washed twice with distilled water, desiccated, and kept at $4^{\circ} \mathrm{C}$ for further characterization.

Table I. Independent Variables (Factors) Used in Formulations and Their Levels

\begin{tabular}{llll}
\hline Code & Factors & \multicolumn{2}{l}{ Levels used } \\
\cline { 3 - 4 } & & Low $(-1)$ & High $(+1)$ \\
\hline$X_{1}$ & Lipid type & Compritol & Precirol \\
$X_{2}$ & Surfactant concentration (\%) & 1 & 2 \\
$X_{3}$ & Cosurfactant concentration (\%) & 0.25 & 0.5 \\
\hline
\end{tabular}

\section{In Vitro Characterization of SLNs}

Determination of Carvedilol Entrapment Efficiency (EE\%)

The amount of entrapped carvedilol was determined by lysis of the separated precipitate $(25 \mathrm{mg})$ in $50 \mathrm{ml}$ of methylene chloride, by centrifugation of the solution at $4000 \mathrm{rpm}$ for $30 \mathrm{~min}$, and then by analyzing the drug content by the HPLC method (13). The analysis was carried out with a liquid chromatograph (Agilent 1260 Infinity, Waldbronn, Germany), equipped with Agilent 1260 Infinity Diode array detector VL (Agilent Technologies, Walnut Creek, CA) (G 1315D), Agilent 1260 Infinity preparative pump (G 1361A), Agilent 1260 Infinity thermostatted column compartment (G 1316A), and Agilent 1260 Infinity preparative auto-sampler (G 2260A).

Separation and quantification were carried out on a C18 column (ZORBAX Eclipse Plus, Agilent Technologies, Walnut Creek, CA) $(25 \mathrm{~cm} \times 4.6 \mathrm{~mm}$ i.d., particle size of $5 \mathrm{~mm})$. The mobile phase was a mixture of acetonitrile/0.05 M disodium hydrogen phosphate $(50: 50, v / v)$ with $\mathrm{pH} 4.0$. The mobile phase was filtered by passing through a $0.45-\mu \mathrm{m}$ membrane filter, degassed, and delivered at a flow rate of $1 \mathrm{ml} / \mathrm{min}$. Detection was performed at a wavelength of $242 \mathrm{~nm}$ at ambient temperature. Carvedilol EE\% was determined using the following formula:

$\mathrm{EE} \%=$ Entrapped Drug $/$ Total drug $\times 100$

\section{Determination of Particle Size and Zeta Potential}

Dynamic light scattering (Zetasizer Nano ZS, Malvern instruments, Malvern, UK) was used to assess the mean particle size, polydispersity index, and zeta potential of SLN dispersions. The samples were diluted with distilled water before measurement. All measurements were performed in triplicate at a temperature of $25 \pm 2^{\circ} \mathrm{C}$ and an angle of $90^{\circ}$ to the incident beam (14).

\section{In Vitro Release Study of Carvedilol-Loaded SLNS}

In vitro release of carvedilol was performed using vertical diffusion Franz cells with an effective diffusion area of $5 \mathrm{~cm}^{2}$. The release studies of carvedilol from SLNs were performed in $50 \mathrm{ml}$ of simulated nasal electrolyte solution (SNES) pH 5.5 (composed of $7.45 \mathrm{mg} / \mathrm{ml} \mathrm{NaCl}, 1.29 \mathrm{mg} / \mathrm{ml} \mathrm{KCl}$, and $0.32 \mathrm{mg}$ / $\mathrm{ml} \mathrm{CaCl} \cdot 2 \mathrm{H}_{2} \mathrm{O}$ and $\mathrm{pH}$ adjusted at 5.5) (15) containing $20 \%$ propylene glycol as receptor medium to ensure sink condition (16).

The aqueous nanoparticulate dispersion equivalent to $3 \mathrm{mg}$ of carvedilol was placed in the donor compartment. The receptor compartment was maintained at $37 \pm 0.5^{\circ} \mathrm{C}$ and stirred by a magnetic bar at $100 \mathrm{rpm}$. The donor compartment was separated from the receptor compartment by cellulose dialyzing membrane with a molecular weight cutoff of $12,000 \mathrm{~g} / \mathrm{mol}$ which was soaked in the receptor medium overnight. At predetermined time intervals $(0.5,1,2,3,4,5,6,7$, and $8 \mathrm{~h}$ ), $1 \mathrm{ml}$ aliquots were withdrawn from the sampling port and were replaced with an equal volume of fresh medium to 
maintain constant volume. The samples were filtered through a $0.45-\mu \mathrm{m}$ pore filter and analyzed by the HPLC method as stated above, and the drug release percent was determined. Plots of percent drug released against time were constructed. The release of the free drug solution $(3 \mathrm{mg}$ carvedilol dissolved in $2 \mathrm{ml}$ ethanol) was also investigated in the same way.

To determine the mechanism of the release of carvedilol from its SLNs, the release data were analyzed using linear regression according to zero-order and first-order as well as Higuchi diffusion model. The correlation coefficient $[r]$ was determined in each case, and accordingly, the orders of release were determined.

\section{Ex Vivo Permeation Study}

Freshly excised sheep nasal mucosa (except the septum part) was collected in phosphate buffer saline (PBS), pH 6.4. The membrane was kept in PBS pH 6.4 for 30 min to equilibrate. The superior nasal concha was identified and separated from the nasal membrane (17). The excised superior nasal membrane was then mounted on Franz diffusion cell. Franz diffusion cell used for ex vivo diffusion studies had a surface area of $5 \mathrm{~cm}^{2}$ and mucosa thickness of $0.2 \pm 0.1 \mathrm{~mm}$. The temperature of the receiver chamber containing $50 \mathrm{ml}$ of diffusion media PBS pH 6.4 containing $20 \%$ propylene glycol was controlled at $37 \pm 0.5^{\circ} \mathrm{C}$ under continuous stirring with a magnetic bar at $100 \mathrm{rpm}$, in a way that the nasal membrane surface just flushes the diffusion fluid. Carvedilol-loaded SLNs (equivalent to $3 \mathrm{mg}$ carvedilol) were placed in the donor compartment of a Franz diffusion cell. Samples from the receptor compartment were withdrawn at predetermined time intervals $(0.5,1,2,3,4,6,8,10,12$, and $24 \mathrm{~h})$, and the receptor chamber was compensated with equal volumes of fresh medium. The withdrawn samples were filtered through a $0.45-\mu \mathrm{m}$ pore filter and finally analyzed by the HPLC method as mentioned before.

The permeation data of carvedilol were graphically plotted as the cumulative amount of drug permeated per unit area as a function of time and the permeation parameters could be provided. The cumulative drug in the receptor compartment after $24 \mathrm{~h}$ was defined as $Q_{24}\left(\mu \mathrm{g} \mathrm{cm}^{-2}\right)$. The permeability coefficient $K_{\mathrm{p}}\left(\mathrm{cm} \mathrm{h}^{-1}\right)$ of carvedilol from each formulation can be calculated by dividing the slope of the straight line portion of the curve (flux) by drug concentration originally added. The lag time could be determined from the X-intercept of the linear portion of the graph.

\section{Optimization Data Analysis and Model Validation}

Data collected from a total of eight runs for each response were fitted to a set of multiple linear regression models using R (version 2.15, R Core Team, Vienna, Austria). The experimental design allows the estimation of a mean term, three main effect terms, three two-way interaction terms, and one three-way interaction term. However, for practical reasons, we assume that the three-way interaction does not exist. This assumption allows us to estimate a residual variability term. To avoid overparameterization, a backward stepwise regression approach was adopted. Starting with a full model, the most insignificant term ( $t$ test, $p$ value $>0.05)$ was eliminated and the reduced model was fitted. This step was done iteratively so that only significant terms remain in the final model.

Evaluation of the final model was based on multiple correlation coefficient $\left(R^{2}\right)$, residual versus fitted values plot, normal quartile-quartile plot, and percentage prediction error (\%PE). In case of poor fitting, the Box-Cox transformation and maximum likelihood was used to estimate a proper power parameter (18).

Our ultimate goal was to develop an optimal formula with high EE\%, small particle size, and large $Q_{24}$. To achieve this goal, a weighted arithmetic mean response $\left(Y_{\text {avg }}\right)$ was calculated by

$Y_{\mathrm{avg}}=\frac{1}{3} Y_{1}^{\prime}+\frac{1}{3} Y_{2}^{\prime}+\frac{1}{3} Y_{3}^{\prime}$

and maximized. $Y_{1}{ }^{\prime}, Y_{2}{ }^{\prime}$, and $Y_{3}{ }^{\prime}$ are the normalized responses. Responses intended for maximization were normalized using the formula:

$$
Y^{\prime}=\frac{Y-Y_{\min }}{Y_{\max }-Y_{\min }}
$$

, while responses intended for minimization were normalized using the formula:

$$
Y^{\prime}=\frac{Y_{\max }-Y}{Y_{\max }-Y_{\min }}
$$

where $Y_{\min }$ and $Y_{\max }$ are the minimum and maximum modelpredicted response.

The equal weights used in Eq. (1) suggest that the three responses had the same importance during optimization.

\section{Confocal Laser Scanning Microscopy (CLSM) Study}

Rhodamine B-labeled SLNs were prepared as described previously with some modifications. A $10-\mathrm{mg} / \mathrm{ml}$ methanol stock solution of rhodamine was prepared. Five microliters from this stock solution was diluted in the organic phase and preparation was continued as mentioned before. CLSM was used to scan the fluorescence signal of labeled SLNs (S8) and methanolic PBS solution of rhodamine $(19,20)$. For CLSM study, the ex vivo permeation study was carried out as described above. After $2 \mathrm{~h}$, the nasal mucosa was removed and washed with distilled water. The treated area was frozen at $-20^{\circ} \mathrm{C}$, and then the frozen mucosa was sectioned with a cryostat into $20 \mu \mathrm{m}$ slices and placed on glass coverslips. The samples were then microscopically examined without additional tissue processing using an inverted laser scanning confocal microscope LSM 710 (Carl Zeiss, Germany). The rhodamine was excited with a $\mathrm{He} / \mathrm{Ne}$ laser (excitation $=$ $524 \mathrm{~nm}$ ) and light emission was detected using a bypass filter $($ emission $=580 \mathrm{~nm})$.

\section{Transmission Electron Microscopy (TEM)}

Formula S8 was selected for morphological examination of the particles. A drop of the diluted emulsion was applied on 
a carbon coated grid. The emulsion was left for 2 min to allow its absorption in the carbon film, and the excess liquid was drawn off with filter paper and an aqueous solution of phosphotungstic acid was used as a negative stain. Samples were examined by TEM (Jeol, Tokyo, Japan) operating at an accelerating voltage of $80 \mathrm{kV}(21)$.

\section{Stability Study of SLNS}

SLN formula S8 was stored in a glass vial at $4{ }^{\circ} \mathrm{C}$ for up to 3 months. Samples from the selected formula were withdrawn at definite time intervals $(30,60$, and 90 days) and characterized for particle size, entrapment efficiency, and zeta potential (21).

\section{Tolerability and Toxicity Studies (Histopathological Studies)}

Four male Wistar albino rats weighing 200-250 g received $20 \mu 1$ of SLNs (S8 after being incorporated into a mucoadhesive in situ gel base which consisted of $20 \%$ poloxamer 407, 10\% poloxamer 188, and 0.5\% Carbopol 971P) once daily via the nasal route for 14 days. The rats were then sacrificed and their nasal septum with the epithelial cell membrane was isolated $(15,22)$, fixed in $10 \%$ formaldehyde for $24 \mathrm{~h}$, decalcified, washed with water, and then dehydrated by ethyl alcohol. Specimens were cleared in xylene then embedded in paraffin at $56^{\circ} \mathrm{C}$ in a hot air oven for $24 \mathrm{~h}$. Tissue blocks of paraffin beeswax were then prepared for sectioning at $5 \mu \mathrm{m}$ by slide microtone. The obtained tissue sections were collected on glass slides, deparaffinized, and stained by hematoxylin and eosin stains. Slides of untreated and treated tissues were examined using a light microscope (23).

\section{Pharmacokinetic Studies}

\section{Animals}

This study was approved by the local animal ethical committee of Beni Suef University. A group of six New Zealand White (NZW) male rabbits weighing $2.5 \pm 0.17 \mathrm{~kg}$ were used in this study. After an overnight fasting, rabbits were dosed, held in restrainers during blood sampling, and were conscious throughout the duration of the experiments.

\section{Study Design}

The study was assigned in a fasted state with a singledose, three-treatment and three-period crossover design with 1 week washout period before administration of another formulation.

\section{Dosage and Drug Administration}

Each rabbit received a dose of $1.0 \mathrm{mg} / \mathrm{kg}$ from each of the three formulations used in this study. The formulations were as follows: carvedilol coarse suspension $(1 \mathrm{mg} / \mathrm{ml})$ in purified water was given by the oral route, in situ gel containing the SLN formula S8 $(12.5 \mathrm{mg} / \mathrm{ml})$ of $100 \mu$ l was administered in each nostril and the carvedilol solution $(1 \mathrm{mg} / \mathrm{ml})$ was prepared in sterile water for injection containing $1 \% \mathrm{w} / \mathrm{v}$ Tween
80 and $2 \% \mathrm{w} / v$ ethanol, and the solution was injected into the marginal ear vein of each rabbit.

\section{Sample Collection}

After administration of the different formulations, $2.0 \mathrm{ml}$ blood samples were collected at different time intervals $(0.5,1$, $2,4,8,12$, and $24 \mathrm{~h}$ ) from the marginal ear vein of each rabbit. Blood samples were collected in EDTA tubes to avoid clotting, and samples were centrifuged at $3000 \mathrm{rpm}$ for 15 min to obtain plasma. The separated plasma tubes were stored at $-20^{\circ} \mathrm{C}$ until assayed.

\section{Chromatographic Conditions}

Plasma samples were analyzed for carvedilol adopting a modified liquid chromatography tandem mass spectrometry (LC-MS/MS) method (24) which consisted of a Shimadzu Prominence (Shimadzu, Japan) series LC system equipped with degasser (DGU-20A3) and auto-sampler (SIL-20 AC) using Luna C18 column (Phenomenex, Inc., Torrance, CA, USA) $(4.6 \times 50 \mathrm{~mm} ; 5 \mu \mathrm{m}$ particle size $)$.

The mobile phase was a mixture of acetonitrile and $0.1 \%$ formic acid $(50: 50 \% \mathrm{v} / \mathrm{v})$ and was delivered at a flow rate of $1 \mathrm{ml} / \mathrm{min}$ into the mass spectrometer's electrospray ionization chamber, and the injection volume was $20 \mu \mathrm{l}$. Quantitation was achieved by MS/MS detection in positive ion mode using a MDS Sciex (Foster City, CA, USA) API-3200 mass spectrometer. The column and auto-sampler tray were maintained at $25^{\circ} \mathrm{C}$. The ion spray voltage was set at $3.6 \mathrm{kV}$ and the nebulizer gas pressure was $35 \mathrm{psi}$. The multiple reaction monitoring (MRM) mode was employed for the quantification: $\mathrm{m} / \mathrm{z}$ $407.2 \rightarrow 100.2$ for carvedilol. Fragmentor and collision energy were adjusted at 145 and $29 \mathrm{~V}$, respectively. Gas temperature was $300^{\circ} \mathrm{C}$ and gas flow rate was $10 \mathrm{l} / \mathrm{min}$. All data acquisition was controlled by Analyst software 1.4.2 (Applied Biosystems Inc., Foster City, CA, USA).

\section{Sample Preparation for Analysis}

An aliquot of $1.5 \mathrm{ml}$ of acetonitrile was added to $0.5 \mathrm{ml}$ of the plasma samples. The samples were vortexed for efficient mixing for $2 \mathrm{~min}$ and centrifuged at $3000 \mathrm{rpm}$ for $10 \mathrm{~min}$, and the clear supernatant was injected into the LC apparatus. The carvedilol recovery $(5-300 \mathrm{ng} / \mathrm{ml})$ varied between 93.65 and $100.26 \%$. The calibration line was linear between 5 and $300 \mathrm{ng} / \mathrm{ml}\left(r^{2}=0.9986\right)(n=8)$.

\section{Data Analysis}

Pharmacokinetic characteristics from plasma data following administration of the three treatments were estimated for each rabbit using WinNonlin ${ }^{\circledR}$ (version 1.5, Scientific Consulting, Inc., USA). Noncompartmental analysis was used. $C_{\max }$ $(\mathrm{ng} / \mathrm{ml})$ and $t_{\max }(\mathrm{h})$ were the observed maximal drug concentration and the time needed to reach this concentration, respectively. The area under the curve, $\mathrm{AUC}_{0-24}(\mathrm{ng} \mathrm{h} / \mathrm{ml})$, was calculated using the trapezoidal rule from zero time to the last time of the blood sample. The AUC from zero to infinity, $\mathrm{AUC}_{0-\infty}(\mathrm{ng} \mathrm{h} / \mathrm{ml})$, was calculated as $\mathrm{AUC}_{0-\infty}=\mathrm{AUC}_{0-24}+$ $C_{t} / k$, where $C_{t}$ is the last measured concentration at time $t$, 
Table II. The $2^{3}$ Factorial Design and the Observed Responses

\begin{tabular}{|c|c|c|c|c|c|c|c|}
\hline \multirow[t]{2}{*}{ Formula } & \multicolumn{3}{|c|}{ Independent variables } & \multicolumn{3}{|l|}{ Dependent variables } & \multirow[t]{2}{*}{ Polydispersity index } \\
\hline & $X_{1}$ (type) & $X_{2}(\% w / v)$ & $X_{3}(\% w / v)$ & $Y_{1}(\%)($ mean $\pm \mathrm{SD})$ & $Y_{2}(\mathrm{~nm})($ mean $\pm \mathrm{SD})$ & $Y_{3}\left(\mu \mathrm{g} / \mathrm{cm}^{2}\right)$ & \\
\hline S1 & Compritol & 1 & 0.25 & $81.31 \pm 1.21$ & $352 \pm 3.49$ & $292.40 \pm 4.20$ & 0.21 \\
\hline S2 & Compritol & 2 & 0.25 & $76.23 \pm 2.33$ & $228 \pm 4.68$ & $369.67 \pm 3.11$ & 0.12 \\
\hline S3 & Compritol & 1 & 0.5 & $87.62 \pm 1.48$ & $209 \pm 3.76$ & $360.78 \pm 6.68$ & 0.17 \\
\hline S4 & Compritol & 2 & 0.5 & $80.07 \pm 2.59$ & $134 \pm 5.11$ & $420.33 \pm 3.42$ & 0.15 \\
\hline S5 & Precirol & 1 & 0.25 & $73.56 \pm 2.34$ & $152 \pm 3.14$ & $387.64 \pm 6.90$ & 0.20 \\
\hline S6 & Precirol & 2 & 0.25 & $68.00 \pm 2.14$ & $89 \pm 5.58$ & $462.85 \pm 5.33$ & 0.11 \\
\hline S7 & Precirol & 1 & 0.5 & $78.41 \pm 1.37$ & $112 \pm 7.88$ & $419.16 \pm 5.67$ & 0.16 \\
\hline S8 & Precirol & 2 & 0.5 & $72.61 \pm 1.09$ & $66 \pm 4.92$ & $551.42 \pm 4.78$ & 0.10 \\
\hline
\end{tabular}

and $k$ is the terminal elimination rate constant estimated by log-linear regression analysis on data visually assessed to be at the terminal log-linear phase. Apparent terminal elimination half-life $\left(t_{1 / 2}\right)$ was calculated as $t_{1 / 2}=0.693 / \mathrm{k}$.

\section{RESULTS AND DISCUSSION}

\section{Carvedilol Entrapment Efficiency}

The EE\% of all SLN formulations is shown in Table II, where $\mathrm{EE} \%$ ranged from $68 \pm 2.14$ to $87.62 \pm 1.48 \%$. The observed high drug EE\% with Compritol and Precirol might be attributed to the high hydrophobicity of the long chain fatty acids attached to the triglycerides leading to enhanced accommodation of lipophilic drugs (25). Compritol and Precirol are complex lipids composed of mixtures of mono-, di-, and triglycerides which form less perfect crystals providing space to accommodate the drugs (26).

SLN formulations containing Compritol (S1-S4) showed a significantly higher $\mathrm{EE} \%$ ( $p$ value $<0.05)$ compared to those containing Precirol (S5-S8). This may be due to the difference in chain length and composition of the used lipids.

It was also clear that increasing the concentration of the surfactant from 1 to $2 \% w / v$ resulted in a significant decrease in the $\mathrm{EE} \%$ of the prepared SLNs $(p$ value $<0.05)$. This decrease in $\mathrm{EE} \%$ could be explained on basis of the partition phenomenon. High surfactant level in the external phase increases solubilization of the drug in this phase resulting in increased partition of the drug from the internal to the external phase (27).
As shown in Table II, increasing lecithin concentration resulted in a consequent increase in $\mathrm{EE} \%$ ( $p$ value $<0.05)$. This could be attributed to the ability of the phospholipid to provide more space to incorporate the drug, and a monomolecular lecithin layer may be formed to stabilize the particle interface (28). This is in accordance with previously reported results $(29,30)$.

\section{Particle Size Analysis}

The particle size of all formulations ranged from $66 \pm 4.92$ to $352 \pm 3.49 \mathrm{~nm}$ with low polydispersity indices indicating narrow particle size distribution (Table II). The results showed that formulations containing Compritol had larger particle sizes than those containing Precirol. This could be attributed to the difference in viscosities and chain lengths of the two lipids (25). Compritol 888 ATO is a solid lipid based on glycerol esters of behenic acid (C22), while Precirol ATO 5 is composed mainly of palmitic (C16) and stearic acid (C18) $>90 \%$. Compritol has a higher melting point $\left(69.0-74.0^{\circ} \mathrm{C}\right)$ than Precirol $\left(50.0-60.0^{\circ} \mathrm{C}\right)$ resulting in higher viscosity in addition to the presence of a long chain hydrocarbon of Compritol which leads to a larger particle size compared to Precirol ( $p$ value $<0.05)$.

There was a decrease in particle size with increasing surfactant concentration ( $p$ value $<0.05$ ). A plausible explanation for this phenomenon is the reduction in interfacial tension between the lipid phase and the aqueous phase leading to the formation of small-sized droplets. Additionally, there is a possibility of the presence of a steric barrier on the surface of the particles produced by a higher surfactant concentration
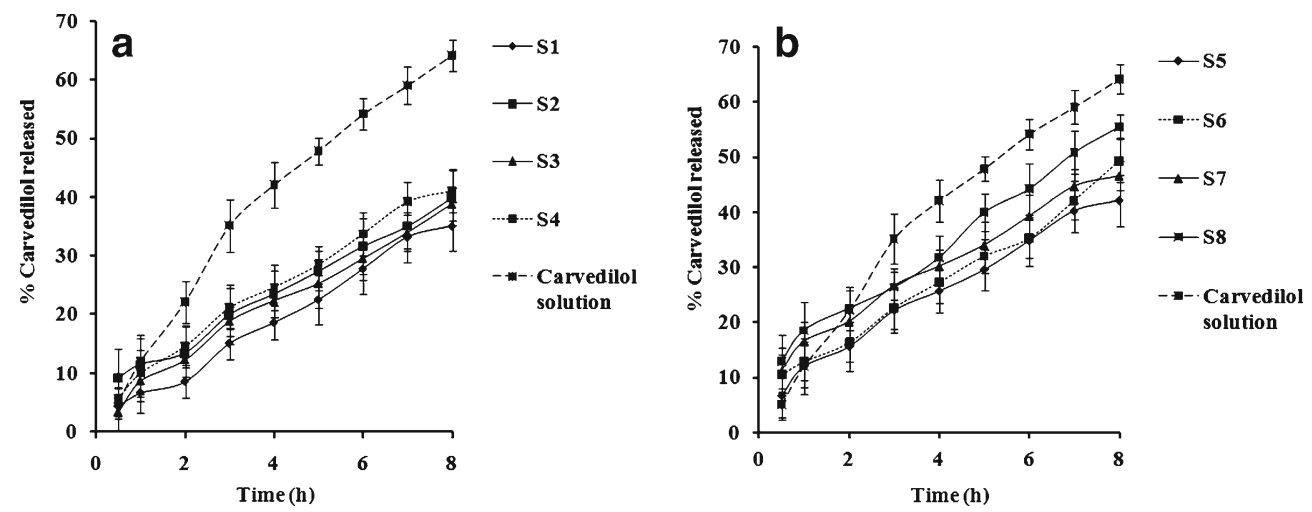

Fig. 1. In vitro release profile of carvedilol from its SLN formulae: a S1-S4 and b S5-S8 
Table III. Ex Vivo Permeation Parameters of Carvedilol-Loaded SLNs Versus Carvedilol Solution

\begin{tabular}{lcc}
\hline Formula no. & Lag time $(\mathrm{min})$ & $K_{\mathrm{p}}\left(\mathrm{cm} \mathrm{h}^{-1}\right)$ \\
\hline S1 & $68 \pm 3.68$ & $0.0140 \pm 0.0051$ \\
S2 & $61 \pm 2.91$ & $0.0163 \pm 0.0043$ \\
S3 & $65 \pm 5.02$ & $0.0160 \pm 0.0089$ \\
S4 & $60 \pm 2.73$ & $0.0182 \pm 0.0033$ \\
S5 & $57 \pm 1.79$ & $0.0191 \pm 0.0024$ \\
S6 & $48 \pm 2.45$ & $0.0203 \pm 0.0039$ \\
S7 & $52 \pm 1.23$ & $0.0198 \pm 0.0063$ \\
S8 & $40 \pm 4.44$ & $0.0234 \pm 0.0013$ \\
Carvedilol solution & $90 \pm 1.62$ & $0.0125 \pm 0.0061$ \\
\hline
\end{tabular}

Data are mean values $(n=3) \pm \mathrm{SD}$

which protects smaller particles and prevents their coalescence into bigger ones (27).

Also, there was a decrease in the particle size upon increasing lecithin concentration $(p$ value $<0.05)$. The increase of lecithin content in SLN formulations could reduce the melting point of the lipid matrix and the interfacial tension between the lipid phase and aqueous phase, hence favoring the formation of SLNs with smaller particle size (31). These findings are in agreement with a previously reported study (32). The combination of co-surfactant and surfactant displayed a synergistic effect and thus decreased the particle size and efficiently prevented particle agglomeration.

Since formulation S8 is Precirol based, it is understandable that its particle size came out smaller than the Compritolbased formulations, S1-S4. Additionally, the higher lecithin and poloxamer 188 content is probably responsible for the smaller particle size of formulation S8 compared to other Precirol-based formulations, S5-S7.

\section{In Vitro Release Study of SLNs}

The release profiles of carvedilol from SLNs as well as the control solution are shown in Fig. 1a, b. Carvedilol-loaded SLN formulations were able to retard its release, and the percentage released up to $8 \mathrm{~h}$ ranged from $35.04 \%$ to $55.51 \%$. Formulations containing Compritol exhibited relatively slower release than those containing Precirol which might be due to the hydrophobic long chain fatty acids of the triglyceride in Compritol that retain the lipophilic drug (33). Moreover, the decreased drug mobility at the experimental release temperature is a result of a higher melting point of Compritol. Paolicelli et al. (34) reported a variation in the release profiles of ibuprofen and acylglycerols with different melting points and suggested that the reason for this variation was the difference in lipid melting points.

Increasing poloxamer 188 concentration from 1 to $2 \%$ $w / v$ led to a corresponding increase in the percentage of carvedilol released which was found for both lipids. The increased surfactant concentration resulted in a higher drug release which may be due to increased partitioning of the drug between the melted lipid phase and the aqueous surfactant phase as a result of greater drug solubility (35).

Increasing lecithin concentration from 0.25 to $0.5 \% \mathrm{w} / \mathrm{v}$ resulted in an increase in the percentage of carvedilol released. A higher release noticed with increasing lecithin concentration could be explained by reducing the interfacial tension between the lipid matrix and aqueous phase and also decreasing the melting point of the lipid matrix as mentioned before. The release profiles of these SLNs behave like the drug-enriched core model (36) where the drug-enriched core is enveloped by a practically drug-free lipid shell. The hindering effects by the surrounding solid lipid shell as well as the increased diffusional distance may result in a retarded drug release profile.

Formula S8 exhibited the highest percentage of carvedilol release which could be attributed to its small size that led to an increase in their surface area. The release pattern of the drug from almost all SLN formulations followed the Higuchi equation with some fitted to zeroorder equation (S4 and S6), while the carvedilol solution followed the first-order kinetics.

\section{Ex Vivo Permeability Study}

Table III shows the calculated permeation parameters for both carvedilol-loaded SLNs and the control solution. The diffusion studies showed that carvedilol-loaded SLNs were able to penetrate the nasal mucosa to a greater extent than
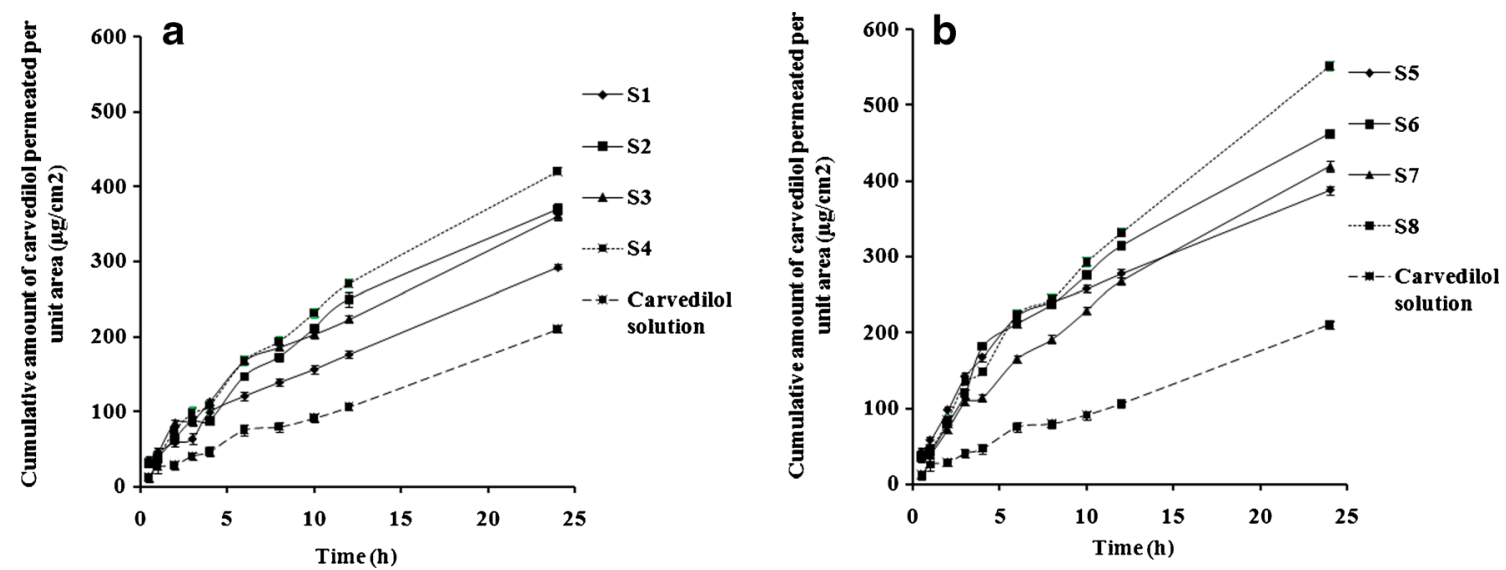

Fig. 2. Permeation profile of carvedilol from its SLN formulae: a S1-S4 and b S5-S8 
Table IV. Analysis of Variance of the Calculated Model for Measured Response

\begin{tabular}{llllll}
\hline Parameters & $D F$ & SS & MS & $F$ & $p$ value \\
\hline EE\% & & & & & \\
$\quad$ Regression & 3 & 253.2 & 84.4 & 187.0 & 0.0001 \\
$\quad$ Residual & 4 & 1.81 & 0.451 & - & - \\
$\quad$ Total & 7 & 255.0 & 255.01 & - & - \\
$\begin{array}{l}\text { Particle size } \\
\quad \text { Regression }\end{array}$ & 3 & 0.009 & 0.003 & 102.9 & 0.0003 \\
$\quad$ Residual & 4 & 0.0001 & 0.0000 & - & - \\
$\quad$ Total & 7 & 0.009 & 0.009 & - & - \\
$Q_{24}$ & & & & & \\
$\quad$ Regression & 3 & 39,875 & 13,292 & 35.38 & 0.002 \\
$\quad$ Residual & 4 & 1503 & 375.7 & - & - \\
$\quad$ Total & 7 & 41,377 & 41,377 & - & - \\
\hline
\end{tabular}

$D F$ degrees of freedom, $S S$ sums of squared error, $M S$ mean squared error $(\mathrm{MS}=\mathrm{SS} / D F), F$ Fisher's ratio $\left(F=\mathrm{MS}_{\text {Regression }} / \mathrm{MS}_{\text {Residual }}\right)$, $E E \%$ entrapment efficiency

the control solution ( $p$ value $<0.05$ ). It seems beneficial to use carvedilol-loaded SLNs rather than the free drug when it comes to intranasal delivery.

This nasal permeation enhancement achieved by the prepared SLNs could be explained by the lipophilic nature of SLNs that may enhance the permeation of the drug and their small particle size, so there are a large surface area and a monolayer film formation that in turn cause intimate drug adhesion with the mucous membrane. Since this monolayer film is hydrophobic, it exhibits an occlusive action that delays the loss of moisture as a result of evaporation, which can enhance the drug permeation (37). Additionally, the crystal modification of the SLN matrix resulting from loss of its water content may induce drug expulsion and penetration (38).

Another reason for this enhancement involves the fact that surfactants can fluidize or loosen the intercellular lipid layer of the nasal mucosa and thus enhance the permeation (39).

a

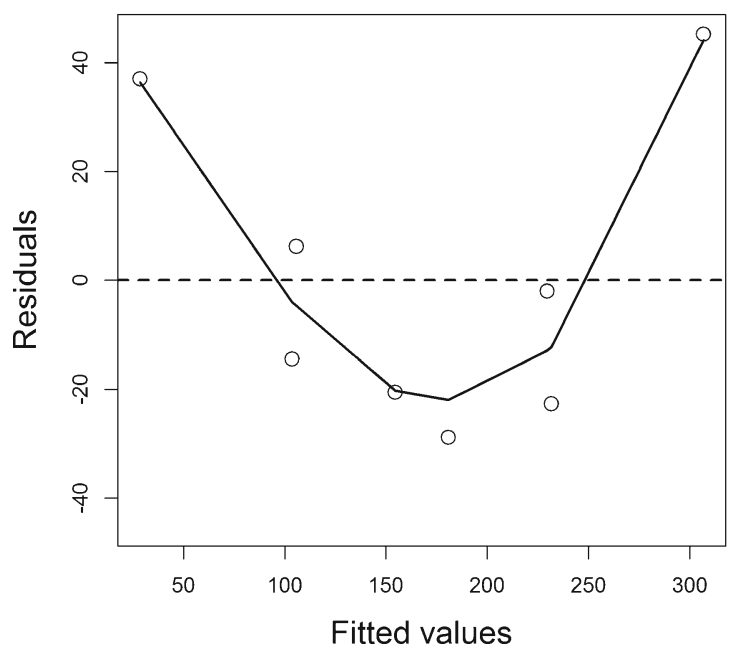

Incorporation of a nonionic surfactant like poloxamer 188 might increase transcellular transport of carvedilol by reducing the barrier function of the mucous layer due to its capability for reducing the mucous viscosity, in addition to tight junctional modulation mediated by the nonionic surfactant (15).

Based on the amount of drug permeated and permeability coefficient, SLNs containing Precirol, a higher concentration of poloxamer $188(2 \% \mathrm{w} / \mathrm{v})$, and a higher concentration of lecithin $(0.5 \% \mathrm{w} / \mathrm{v})$ exhibited enhanced delivery of carvedilol through the nasal mucosa. These results correlate well with in vitro drug release results. The results obtained were presented in Fig. 2a, b.

Formula S8 (containing Precirol, 2\% $w / v$ poloxamer 188 , and $0.5 \% w / v$ lecithin) exhibited the highest permeability coefficient, the highest amount of permeated drug, and the shortest lag time; these results could be attributed to its smallest particle size and lowest polydispersity index which gave the highest surface area and the lowest aggregation of the nanoparticles that might improve the diffusion of the drug. Also the small particle size increases the occlusive effect and so the smallest particle size will give the greatest occlusion which might lead to a valuable increase in diffusion. The lowest permeation parameters were obtained in formula S1 (containing Compritol, 1\% $w / v$ poloxamer 188 , and $0.25 \% w / v$ lecithin) which had the largest particle size. These results are in agreement with what has been reported in the literature (40).

\section{Experimental Design and Regression Analysis}

A total of eight experiments representing all combinations of low and high levels of the three formulation factors were simultaneously fitted to multiple linear regression models. Stepwise regression analysis indicated that none of the factor interaction terms was significant for all of the responses; therefore, they were excluded from the final models. b

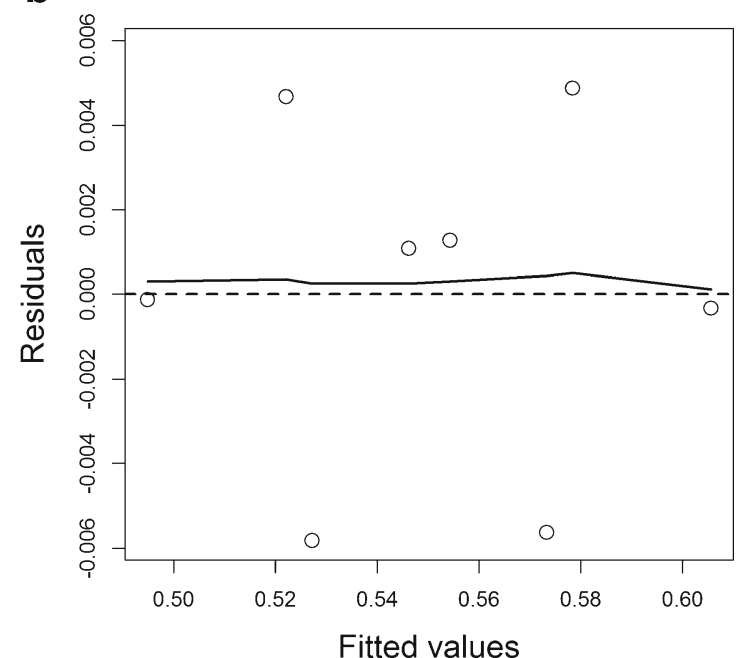

Fig. 3. a Residuals versus fitted values plots for the linear model and $\mathbf{b}$ power-transformed model. The solid line is a LOWESS smother 
a

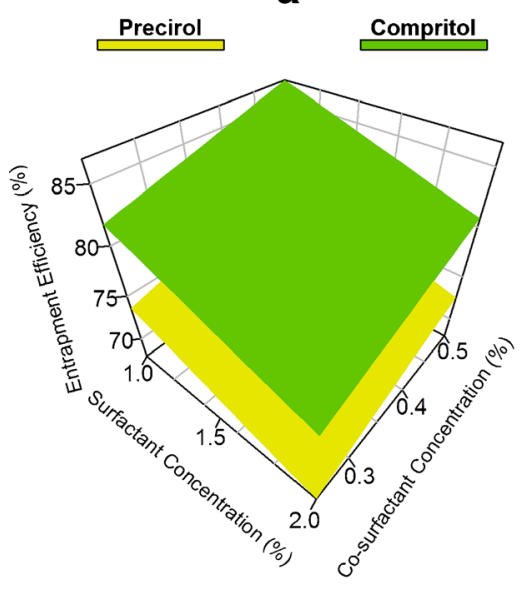

b

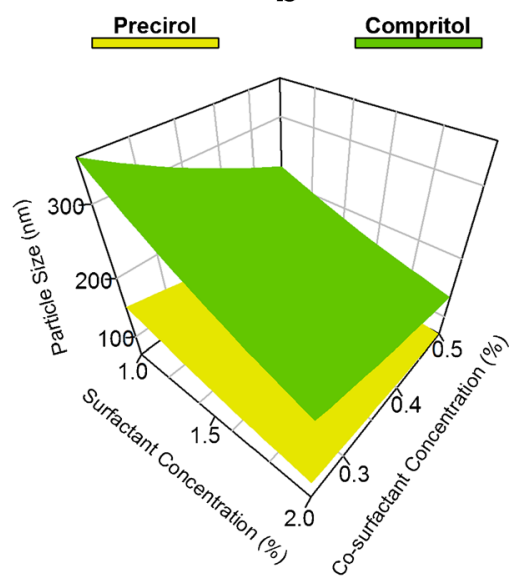

c

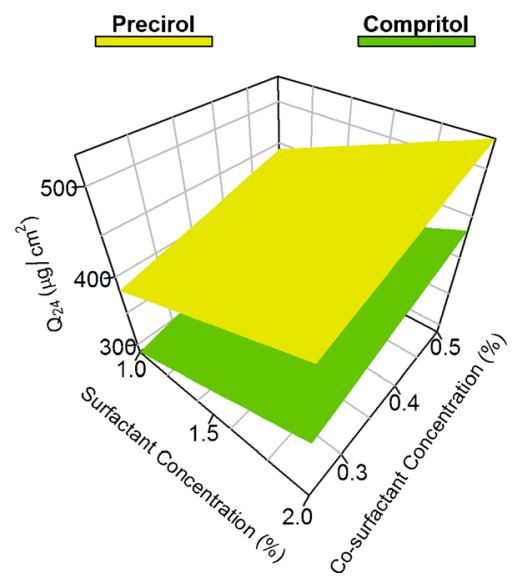

Fig. 4. Response surface plots showing the effect of lipid type, surfactant, and co-surfactant concentrations on a entrapment efficiency, b particle size, and c $Q_{24}$

The analysis of variance of the final models for the responses $Y_{1}(\mathrm{EE} \%), Y_{2}$ (particle size), and $Y_{3}\left(Q_{24}\right)$ depicted in Table IV demonstrates that the models explained a significant proportion of the variability in the observed responses ( $F$ ratio test, $p$ value $<0.01$ ). $R^{2}$ for $Y_{1}$ and $Y_{3}$ were 0.99 and 0.96 , respectively, suggesting that the final models could satisfactorily account for the variability in these responses.

Residual plots for $Y_{1}$ and $Y_{2}$ (not shown) did not show clear patterns indicating a lack of prediction bias. Fitting a linear regression model to $Y_{2}$ failed to achieve an adequate fit, as indicated by $R^{2}$ of 0.90 , nonlinear residual plot (Fig. 3a), and \%PE up to $57 \%$. Using the Box-Cox transformation and maximum likelihood, a power of -0.12 was deemed appropriate for $Y_{2}$ variable transformation. Power transformation of $Y_{2}$ resulted in $R^{2}$ of 0.99 , random distribution of residuals around the zero line (Fig. $3 \mathrm{~b}$ ), and \%PE up to $9 \%$ (Table V). Quartile-quartile plots (not shown) indicated that the normality assumption was satisfied by all models.

The regression analysis proposes the following equations:

$Y_{1}=77.23-4.08 \cdot X_{1}-3.00 \cdot X_{2}+2.45 \cdot X_{3}$

$Y_{2}^{-0.12}=0.550+0.026 \cdot X_{1}+0.016 \cdot X_{2}+0.014 \cdot X_{3}$
$Y_{3}=407.9+47.30 \cdot X_{1}+43.12 \cdot X_{2}+29.80 \cdot X_{3}$

Equations 4-6 suggest that formulations made using Precirol $\left(X_{1}=+1\right)$ exhibit lower entrapment efficiency, smaller particle size, but larger $Q_{24}$, compared to Compritol-based formulations $\left(X_{1}=-1\right)$. Surfactant concentration $\left(X_{2}\right)$ has a negative effect on entrapment efficiency and particle size (Eqs. 4 and 5). Concentration of the co-surfactant $\left(X_{3}\right)$ has a positive effect on entrapment efficiency (Eq. 4), but a negative effect on particle size (Eq. 5). Increasing surfactant and co-surfactant concentrations is associated with higher $Q_{24}$ values (Eq. 6).

\section{Response Surface Analysis}

Three-dimensional response surface visualization of the interactive effects of surfactant concentration and co-surfactant concentration at the two levels of lipid type is depicted in Fig. 4. As can be seen, response surfaces for EE\% (Fig. 4a) and $Q_{24}$ (Fig. 4c) were perfectly linear and nearly parallel between Compritol and Precirol. This finding suggests the lack of interaction between levels of all factors in these responses. For particle size, Compritol and Precirol surfaces showed slight deviation from parallel behavior 
Table V. The Observed and Model-Predicted Response Values and Prediction Error

\begin{tabular}{|c|c|c|c|c|}
\hline Formulation & Response & $\begin{array}{l}\text { Observed } \\
\text { value }\end{array}$ & $\begin{array}{l}\text { Predicted } \\
\text { value }\end{array}$ & $\% \mathrm{PE}$ \\
\hline S1 & \multirow[t]{8}{*}{$Y_{1}$} & 81.31 & 81.85 & -0.669 \\
\hline S2 & & 76.23 & 75.86 & 0.490 \\
\hline S3 & & 87.62 & 86.76 & 0.985 \\
\hline S4 & & 80.07 & 80.76 & -0.865 \\
\hline S5 & & 73.56 & 73.69 & -0.181 \\
\hline S6 & & 68.00 & 67.70 & 0.447 \\
\hline S7 & & 78.41 & 78.60 & -0.237 \\
\hline S8 & & 72.61 & 72.60 & 0.021 \\
\hline S1 & \multirow[t]{8}{*}{$Y_{2}$} & 352.0 & 351.2 & 0.216 \\
\hline $\mathrm{S} 2$ & & 227.7 & 207.5 & 8.840 \\
\hline S3 & & 209.0 & 225.1 & -7.711 \\
\hline S4 & & 134.0 & 136.6 & -1.937 \\
\hline S5 & & 152.0 & 154.5 & -1.664 \\
\hline S6 & & 89.33 & 95.80 & -7.235 \\
\hline S7 & & 112.0 & 103.2 & 7.893 \\
\hline S8 & & 65.67 & 65.38 & 0.444 \\
\hline S1 & \multirow[t]{8}{*}{$Y_{3}$} & 292.4 & 287.7 & 1.610 \\
\hline S2 & & 369.6 & 373.9 & -1.173 \\
\hline S3 & & 360.1 & 347.3 & 3.566 \\
\hline S4 & & 420.3 & 433.5 & -3.144 \\
\hline S5 & & 387.6 & 382.3 & 1.369 \\
\hline S6 & & 462.8 & 468.5 & -1.226 \\
\hline S7 & & 419.0 & 441.9 & -5.454 \\
\hline S8 & & 551.4 & 528.1 & 4.212 \\
\hline
\end{tabular}

$\% P E$ percentage prediction error $(\% \mathrm{PE}=($ Observed - Predicted $) /$ Observed $\times 100 \%$ )

(Fig. 4b). Increasing surfactant and co-surfactant concentrations produced a more prominent reduction in particle size when Compritol, rather than Precirol, was used as the formulating lipid. Unlike entrapment efficiency and $Q_{24}$, the surface of particle size is nonlinear showing a quadratic-like path as the co-surfactant concentration increases from 0.25 to 0.4 , then the surface levels off. At both lipid types, the sides of the surface were not exactly parallel. Increasing co-surfactant concentration from 0.25 to 0.5 decreases the particle size approximately from 350 to $225 \mathrm{~nm}$ at a constant concentration of the surfactant $(1 \%)$. At $2 \%$ surfactant, particle size decreases approximately from 200 to $130 \mathrm{~nm}$ as the co-
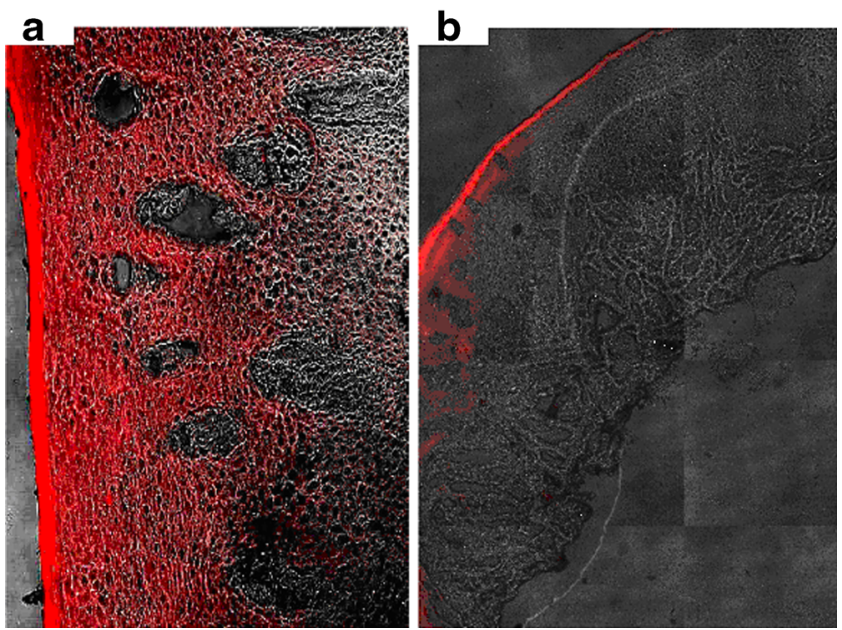

Fig. 5. Confocal images of cross sections of nasal mucosa after application of a rhodamine-loaded SLNs (S8) and $\mathbf{b}$ rhodamine solution

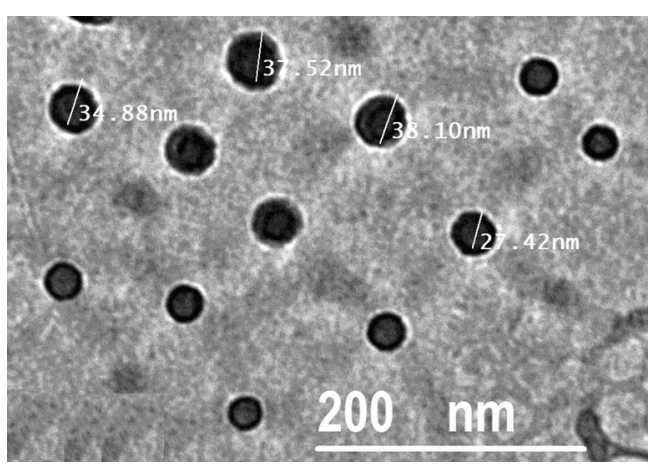

Fig. 6. Transmission electron micrograph of formula S8

surfactant concentration increases. This finding is indicative of a possible positive interaction between surfactant and co-surfactant concentrations, even though this interaction turned out to be statistically insignificant.

\section{Model Validation and Formulation Optimization}

The results of the experiments, model simulated values, as well as \% PE of responses $Y_{1}, Y_{2}$, and $Y_{3}$ for all formulations are listed in Table $\mathrm{V}$. The developed models reasonably predicted the responses with \%PE less than 1,9 , and $6 \%$ for $Y_{1}, Y_{2}$, and $Y_{3}$, respectively. The adequate predictive performance of the models justifies their application in the rationalization of an optimal formula.

According to Table V, formula S3 has the highest EE\%, while formula S8 has the smallest particle size and largest $Q_{24}$ value. Our developed models, combined with a weighted mean response approach, were used to rationalize an optimum formula that would have the largest $\mathrm{EE} \%$, smallest particle size, and highest $Q_{24}$ values possible. Formulation S8 with Precirol, 2\% surfactant, and $0.5 \%$ co-surfactant fulfilled these criteria.

\section{Confocal Laser Scanning Microscopy Study}

In order to visualize the nasal delivery of the prepared SLNs, the optimized formula S8 was considered in this study. It was made fluorescent by the inclusion of rhodamine B (a lipophilic dye) in the lipid phase. The results showed an increased nasal mucosa penetration when rhodamine B was loaded in SLNs (S8), compared to the aqueous solution as shown in Fig. 5. The fluorescent solution was visualized only up to the upper layers, while SLNs were assessed up to the deep layers of the nasal mucosa with a high fluorescence intensity and homogeneous distribution, evidencing the potential of SLNs as a penetration enhancer in nasal drug delivery.

\section{Transmission Electron Microscopy}

TEM was conducted to investigate the shape and surface morphology of carvedilol-loaded SLNs. From Fig. 6, the TEM micrograph of S8 revealed that nanoparticles were almost spherical with smooth morphology and uniform distribution.

There are three different models supporting drug distribution theory within SLNs: (1) a core-shell model with a drugenriched core, (2) a core-shell model with a drug-enriched 


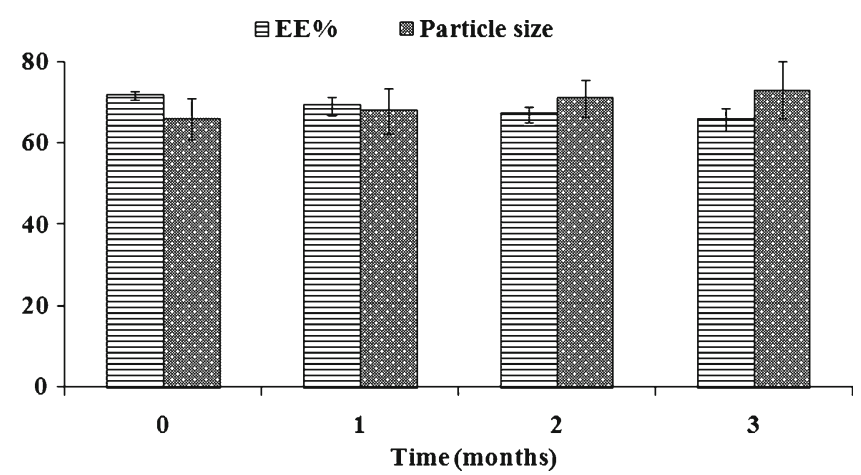

Fig. 7. Effect of storage on the amount of carvedilol retained and particle size of SLN formula S8

shell, and (3) a solid solution model (26). Figure 6 illustrates the appearance of a thin layer surrounding the particles which postulate a drug-enriched core model. This model can be obtained if the drug precipitates first during the lipid solidification process which results in a drug-enriched core covered with a lipid shell with a decreased drug concentration.

\section{Stability Study of SLNs}

The stability of the optimized formula $\mathrm{S} 8$ was studied for $\mathrm{EE} \%$, particle size, and zeta potential after 3 months of storage at $4{ }^{\circ} \mathrm{C}$. As illustrated in Fig. 7, formula S8 showed an insignificant change in $\mathrm{EE} \%$ and particle size during the period of study ( $p$ value $>0.05$ ).

Formula S8 was negatively charged where the zeta potential was found to be $-30.6 \pm 1.9 \mathrm{mV}$ indicating a dispersion quality. Formula S8 showed an insignificant change in zeta potential upon storage indicating good stability ( $p$ value $>$ 0.05 ) (Fig. 8). Zeta potential is considered as a valuable marker for the stability of colloidal dispersions, as it significantly determines the extent of electrostatic repulsion between all adjacent, similarly charged particles in the dispersion. Consequently, the dispersions with high zeta potential are electrically more stable than those with low zeta potentials. The zeta potential value of formulation S8 was far from the aggregation threshold defined by Riddick (41), which confirms its stability. Moreover, the high stability of formula S8 could be attributed to the presence of a decreased amount of partial glycerides like monoglycerides (8-22\%) in Precirol (25).

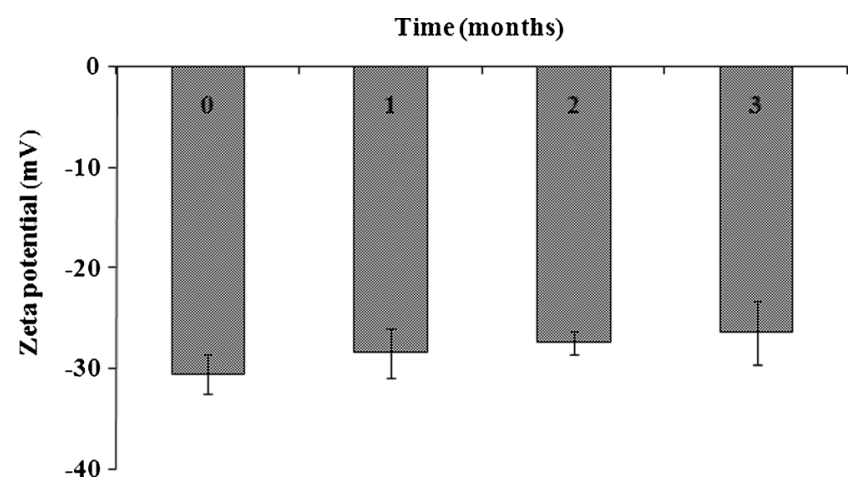

Fig. 8. Effect of storage on zeta potential of SLN formula S8
The improved stability observed ensures the importance of surfactant and co-surfactant combination. Poloxamer 188 has been reported to be one of the most effective nonionic surfactants to prevent the formation of aggregates (42), through steric stabilization of the formed emulsion and formation of a dense surfactant film at the particle/water interface leading to increased zeta potential of the formulation (more negative) (43). Moreover, additional stabilization of

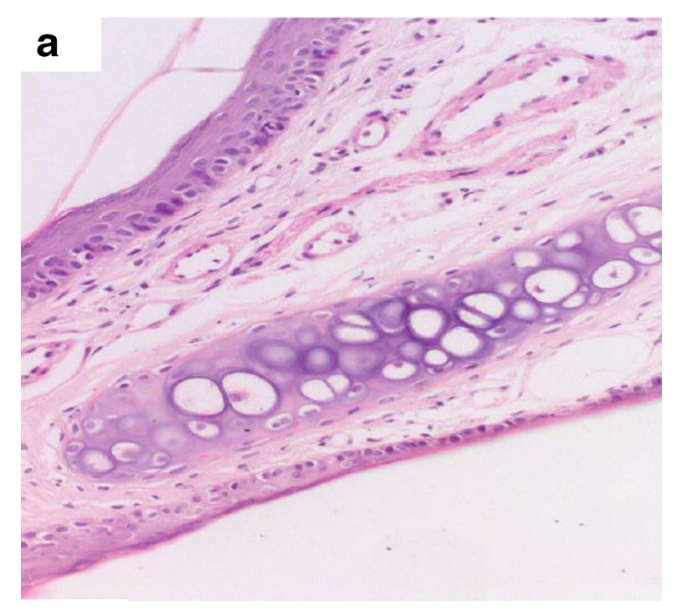

b

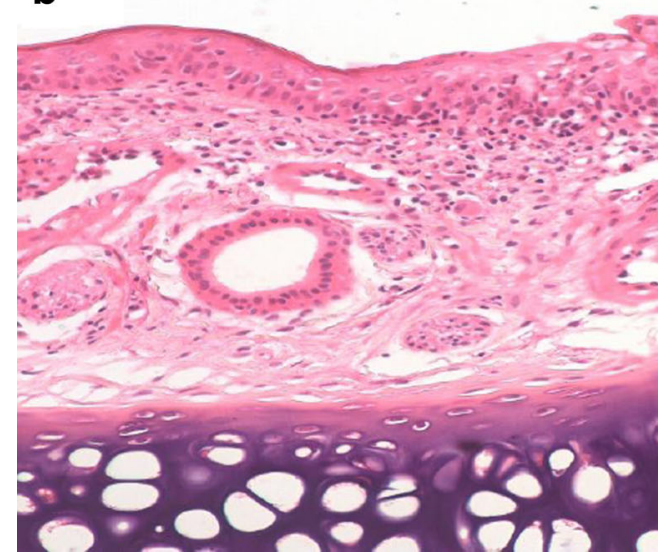

Fig. 9. Light photomicrograph of a untreated rat epithelium b rat epithelium treated with in situ gelling of carvedilol-loaded SLN formula $\mathrm{S} 8$ 


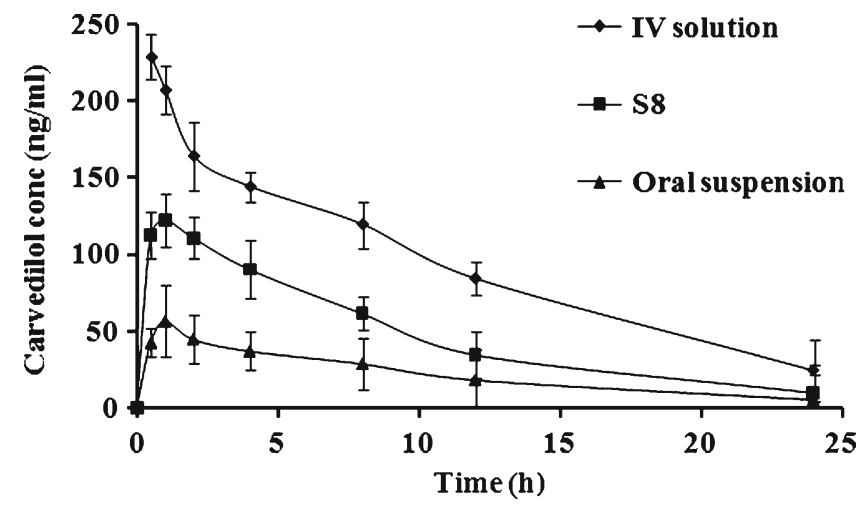

Fig. 10. Mean carvedilol concentrations in plasma of rabbits after administration of IV solution, in situ gelling SLN formula S8, and oral suspension

the emulsion may be provided by lecithin via electrostatic repulsion since it is an amphoteric surfactant (44).

\section{Tolerability and Toxicity Studies (Histopathological Studies)}

Tolerability and toxicity of intranasal formulations are essential parameters that should be assessed. Figure 9 shows light photomicrographs taken from anterior cross sections of rat nasal cavity following 14 days of exposure to in situ gelling SLN formula S8. Examination showed that none of the severe signs such as the appearance of necrosis, sloughing of epithelial cells, or hemorrhage was detected in any of the rats. However, minimal degenerative changes in the olfactory epithelium in addition to a mild inflammatory reaction in the lamina propria were observed.

\section{Pharmacokinetic Studies}

The pharmacokinetic parameters of carvedilol in rabbit plasma following administration of in situ gelling SLN formula $\mathrm{S} 8$, intravenous solution, and oral coarse suspension were assessed using the LC-MS/MS method. The LC-MS/MS assay was validated and confirmed good linearity from 5 to $300 \mathrm{ng} /$ $\mathrm{ml}$ with acceptable intraday and interday reproducibility. The mean plasma drug concentration-time profiles after administration of the different formulations are illustrated in Fig. 10.
Maximum drug concentration was attained after $1 \mathrm{~h}$ following administration of the intranasal in situ gelling SLN formula S8 and the oral suspension.

Table VI shows the pharmacokinetic data for the different formulations. The $C_{\max }$ was $122.49 \pm 16.17 \mathrm{ng} / \mathrm{ml}$ for formula S8, while it was $57.02 \pm 4.38 \mathrm{ng} / \mathrm{ml}$ for the oral suspension indicating higher plasma drug levels in case of the nasal formula S8 as compared to the oral drug suspension ( $p$ value $<0.05$ ). Additionally, the $\mathrm{AUC}_{0-\infty}$ values were $2496.86,1263.83$, and $601.21 \mathrm{ng} \mathrm{h} / \mathrm{ml}$ for the IV solution, the nasal formula S8, and the oral drug suspension, respectively. The superior absolute bioavailability of the nasal formula S8 $(50.63 \%)$ compared to the oral suspension $(24.11 \%)$ clearly indicates a higher absorption of carvedilol when administered intranasally. This enhancement in bioavailability achieved by in situ gelling SLN formula S8 compared to the oral route is attributed to avoiding the first-pass metabolism attendant with peroral drug administration and increased permeability of SLN formulation.

The bioavailability results were consistent with a previous report evaluating a formulation of carvedilol-loaded nanotransfersomes developed by our group (45) and also carvedilol nanosuspension loaded into an in situ gelling nasal spray (46). This finding suggests the necessity of implementing nanotechnology for the successful systemic delivery of carvedilol across the nasal mucosa.

Carvedilol-loaded SLNs were not administered in plain form to rabbits; instead, these SLNs were incorporated in a gel for nasal administration. Using a gel base was crucial to avoid nasal mucociliary clearance in order to achieve reasonable residence time. Although gel formulation is expected to influence drug flux across the nasal mucosa through mucoadhesion, this was not the case in our study. Ex vivo permeation of carvedilol using the sheep model did not improve by incorporating the free drug in the gel formulation ( $p$ value $>0.05$, data not shown). This finding suggests that the improvement in bioavailability seen in our study is attributed solely to the SLN formulation.

\section{CONCLUSION}

We successfully optimized a SLN formulation for intranasal delivery of carvedilol. The formulation consists

Table VI. Pharmacokinetic Parameters for Carvedilol in Rabbit Plasma Following Administration of Intravenous Solution, Intranasal In Situ Gelling SLN Formula S8, and Oral Suspension

\begin{tabular}{llll}
\hline Pharmacokinetic parameter & \multicolumn{2}{l}{ Mean $\pm \mathrm{SD}$} & \\
\cline { 2 - 4 } & Intravenous solution & Intranasal in situ gelling SLN formula S8 & Oral suspension \\
\hline$C_{\max }(\mathrm{ng} / \mathrm{ml})$ & $228.36 \pm 16.94$ & $122.49 \pm 16.17$ & $57.02 \pm 4.38$ \\
$t_{\max }(\mathrm{h})$ & 0.5 & 1 & 1 \\
$K_{\text {elim }}\left(\mathrm{h}^{-1}\right)$ & $0.0997 \pm 0.0236$ & $0.1091 \pm 0.0110$ & $0.1010 \pm 0.0080$ \\
$t_{1 / 2}(\mathrm{~h})$ & $7.00 \pm 1.79$ & $6.35 \pm 0.69$ & $6.86 \pm 0.72$ \\
$\operatorname{AUC}_{0-24}(\mathrm{ng} \mathrm{h} / \mathrm{ml})$ & $2249.74 \pm 275.79$ & $1170.29 \pm 325.28$ & $544.47 \pm 133.68$ \\
$\operatorname{AUC}_{0-\infty}(\mathrm{ng} \mathrm{h} / \mathrm{ml})$ & $2496.86 \pm 391.21$ & $1263.83 \pm 373.39$ & $601.21 \pm 147.88$ \\
$F_{\text {abs }}(\%)$ & 100 & $50.63 \pm 11.25$ & $24.11 \pm 6.75$ \\
\hline
\end{tabular}


of $100 \mathrm{mg}$ Precirol, $2 \%(w / v)$ poloxamer 188 as a surfactant, and $0.5 \%(w / v)$ lecithin as a co-surfactant. Our investigation shows that the SLN formulation facilitates permeation of large carvedilol quantities across the nasal mucosa and, hence, produces a significantly larger absolute bioavailability as compared to an orally administered drug.

\section{ACKNOWLEDGMENTS}

The authors would like to thank Prof. Dr. Mahmoud Al Begawy and Dr. El Shaymaa Al-Nahass, Department of Pathology, Faculty of Veterinary Medicine, Beni-Suef University for helping in histopathological studies and CLSM studies. We also are grateful to Dr. Reda Abd Allah, Department of Anatomy and Embryology, Faculty of Veterinary Medicine, Beni-Suef University for helping in trimming nasal mucosa for ex vivo permeability studies and Dr. Ossama M. Sayed, Department of Pharmaceutics and Industrial Pharmacy, Faculty of Pharmacy, Beni-Suef University for helping in the analysis of plasma samples.

\section{COMPLIANCE WITH ETHICAL STANDARDS}

\section{Conflict of Interest}

competing interests.

The authors declare that they have no

\section{REFERENCES}

1. Packer M, Fowler MB, Roecker EB, Coats AJS, Katus HA, Krum $\mathrm{H}$, et al. Effect of carvedilol on the morbidity of patients with severe chronic heart failure results of the carvedilol prospective randomized cumulative survival (COPERNICUS) study. Circulation. 2002;106:2194-9.

2. Müller RH, Runge SA, Ravelli V, Thünemann AF, Mehnert W, Souto EB. Cyclosporine-loaded solid lipid nanoparticles (SLN): drug-lipid physicochemical interactions and characterization of drug incorporation. Eur J Pharm Biopharm. 2008;68:535-44.

3. Kaur IP, Bhandari R, Bhandari S, Kakkar V. Potential of solid lipid nanoparticles in brain targeting. J Control Release. 2008;127:97-109.

4. Venkateswarlu V, Manjunath K. Preparation, characterization and in vitro release kinetics of clozapine solid lipid nanoparticles. J Control Release. 2004;95:627-38.

5. Bargoni A, Cavalli R, Caputo O, Fundarò A, Gasco MR, Zara GP. Solid lipid nanoparticles in lymph and plasma after duodenal administration to rats. Pharm Res. 1998;15:745-50.

6. Cavalli R, Bargoni A, Podio V, Muntoni E, Zara GP, Gasco MR. Duodenal administration of solid lipid nanoparticles loaded with different percentages of tobramycin. J Pharm Sci. 2003;92:1085-94.

7. Venishetty VK, Chede R, Komuravelli R, Adepu L, Sistla R, Diwan PV. Design and evaluation of polymer coated carvedilol loaded solid lipid nanoparticles to improve the oral bioavailability: a novel strategy to avoid intraduodenal administration. Colloids Surf B: Biointerfaces. 2012;95:1-9.

8. Illum L. Nasal drug delivery-possibilities, problems and solutions. J Control Release. 2003;87:187-98.

9. Manjunath K, Venkateswarlu V. Pharmacokinetics, tissue distribution and bioavailability of clozapine solid lipid nanoparticles after intravenous and intraduodenal administration. J Control Release. 2005;107:215-28.

10. Huang G, Zhang N, Bi X, Dou M. Solid lipid nanoparticles of temozolomide: potential reduction of cardial and nephric toxicity. Int J Pharm. 2008;355:314-20.
11. Patel S, Chavhan S, Soni H, Babbar AK, Mathur R, Mishra AK, et al. Brain targeting of risperidone-loaded solid lipid nanoparticles by intranasal route. J Drug Target. 2011;19:468-74.

12. Trotta M, Debernardi F, Caputo O. Preparation of solid lipid nanoparticles by a solvent emulsification-diffusion technique. Int J Pharm. 2003;257:153-60.

13. Haggag RS, Shaalan RA, Belal TS. Validated HPLC determination of the two fixed dose combinations (chlordiazepoxide hydrochloride and mebeverine hydrochloride; carvedilol and hydrochlorothiazide) in their tablets. J AOAC Int. 2010;93:1192-200.

14. Shiva G, Somaye M, Reza JM. Improved photostability, reduced skin permeation and irritation of isotretinoin by solid lipid nanoparticles. Acta Pharm. 2012;62:547-62.

15. Zaki NM, Awad GA, Mortada ND, Abd ElHady SS. Enhanced bioavailability of metoclopramide $\mathrm{HCl}$ by intranasal administration of a mucoadhesive in situ gel with modulated rheological and mucociliary transport properties. Eur J Pharm Sci. 2007;32:296-307.

16. Aboelwafa AA, El-Setouhy DA, Elmeshad AN. Comparative study on the effects of some polyoxyethylene alkyl ether and sorbitan fatty acid ester surfactants on the performance of transdermal carvedilol proniosomal gel using experimental design. AAPS Pharm Sci Technol. 2010;11:1591-602.

17. Gavini E, Rassu G, Sanna V, Cossu M, Giunchedi P. Mucoadhesive microspheres for nasal administration of an antiemetic drug, metoclopramide: in-vitro/ex-vivo studies. J Pharm Pharmacol. 2005;57:287-94.

18. Venables WN, Ripley BD. Modern applied statistics with S. New York: Springer; 2002.

19. Marttin E, Verhoef JC, Cullander C, Romeijn SG, Nagelkerke JF, Merkus FWHM. Confocal laser scanning microscopic visualization of the transport of dextrans after nasal administration to rats: effects of absorption enhancers. Pharm Res. 1997;14:631-7.

20. Ghirardelli R, Bonasoro F, Porta C, Cremaschi D. Identification of particular epithelial areas and cells that transport polypeptidecoated nanoparticles in the nasal respiratory mucosa of the rabbit. Biochim Biophys Acta. 1999;1416:39-47.

21. Liu J, Hu W, Chen H, Ni Q, Xu H, Yang X. Isotretinoin-loaded solid lipid nanoparticles with skin targeting for topical delivery. Int J Pharm. 2007;328:191-5.

22. Tengamnuay P, Sahamethapat A, Sailasuta A, Mitra AK. Chitosans as nasal absorption enhancers of peptides: comparison between free amine chitosans and soluble salts. Int J Pharm. 2000;197:53-67.

23. Bancroft JD, Gamble M. Theory and practice of histological techniques. Elsevier Health Sciences; 2008.

24. Kim S-H, Lee SH, Lee HJ. Rapid and sensitive carvedilol assay in human plasma using a high-performance liquid chromatography with mass/mass spectrometer detection employed for a bioequivalence study. AJAC. 2010;1:135.

25. Jenning V, Gohla S. Comparison of wax and glyceride solid lipid nanoparticles (SLN $®$ ). Int J Pharm. 2000;196:219-22.

26. Müller RH, Mäder K, Gohla S. Solid lipid nanoparticles (SLN) for controlled drug delivery-a review of the state of the art. Eur J Pharm Biopharm. 2000;50:161-77.

27. Rahman Z, Zidan AS, Khan MA. Non-destructive methods of characterization of risperidone solid lipid nanoparticles. Eur J Pharm Biopharm. 2010;76:127-37.

28. Schubert MA, Müller-Goymann CC. Characterisation of surface-modified solid lipid nanoparticles (SLN): influence of lecithin and nonionic emulsifier. Eur J Pharm Biopharm. 2005;61:77-86.

29. Shah KA, Date AA, Joshi MD, Patravale VB. Solid lipid nanoparticles (SLN) of tretinoin: potential in topical delivery. Int J Pharm. 2007;345:163-71.

30. Liu D, Jiang S, Shen H, Qin S, Liu J, Zhang Q, et al. Diclofenac sodium-loaded solid lipid nanoparticles prepared by emulsion/ solvent evaporation method. J Nanoparticle Res. 2011;13:237586.

31. You J, Wan F, de Cui F, Sun Y, Du Y-Z. Preparation and characteristic of vinorelbine bitartrate-loaded solid lipid nanoparticles. Int J Pharm. 2007;343:270-6.

32. Jenning V, Mäder K, Gohla SH. Solid lipid nanoparticles (SLN) based on binary mixtures of liquid and solid lipids: a (1)H-NMR study. Int J Pharm. 2000;205:15-21. 
33. Kumar VV, Chandrasekar D, Ramakrishna S, Kishan V, Rao YM, Diwan PV. Development and evaluation of nitrendipine loaded solid lipid nanoparticles: influence of wax and glyceride lipids on plasma pharmacokinetics. Int J Pharm. 2007;335:167-75

34. Paolicelli P, Cerreto F, Cesa S, Feeney M, Corrente F, Marianecci $\mathrm{C}$, et al. Influence of the formulation components on the properties of the system SLN-dextran hydrogel for the modified release of drugs. J Microencapsul. 2009;26:355-64.

35. Zur Mühlen A, Schwarz C, Mehnert W. Solid lipid nanoparticles (SLN) for controlled drug delivery-drug release and release mechanism. Eur J Pharm Biopharm. 1998;45:149-55.

36. Wissing SA, Kayser O, Müller RH. Solid lipid nanoparticles for parenteral drug delivery. Adv Drug Deliv Rev. 2004;56:1257-72.

37. Wissing SA, Müller RH. The influence of solid lipid nanoparticles on skin hydration and viscoelasticity-in vivo study. Eur J Pharm Biopharm. 2003;56:67-72.

38. Teeranachaideekul V, Boonme P, Souto EB, Müller RH, Junyaprasert VB. Influence of oil content on physicochemical properties and skin distribution of Nile red-loaded NLC. J Control Release. 2008;128:134-41.

39. Fang J-Y, Fang C-L, Liu C-H, Su Y-H. Lipid nanoparticles as vehicles for topical psoralen delivery: solid lipid nanoparticles
(SLN) versus nanostructured lipid carriers (NLC). Eur J Pharm Biopharm. 2008;70:633-40.

40. Wissing SA, Müller RH. Solid lipid nanoparticles as carrier for sunscreens: in vitro release and in vivo skin penetration. J Control Release. 2002;81:225-33.

41. Riddick TM. Control of colloid stability through zeta potential: with a closing chapter on its relationship to cardiovascular disease. Philadelphia: Livingston Publishing Company; 1968.

42. Liu F, Yang J, Huang L, Liu D. New cationic lipid formulations for gene transfer. Pharm Res. 1996;13:1856-60.

43. Schwarz C, Mehnert W. Solid lipid nanoparticles (SLN) for controlled drug delivery II. Drug incorporation and physicochemical characterization. J Microencapsul. 1999;16:205-13.

44. Müller RH, Jacobs C. Buparvaquone mucoadhesive nanosuspension: preparation, optimisation and long-term stability. Int J Pharm. 2002;237:151-61.

45. Aboud HM, Ali AA, El-Menshawe SF, Elbary AA. Nanotransfersomes of carvedilol for intranasal delivery: formulation, characterization and in vivo evaluation. Drug Deliv. 2015; 1 11 .

46. Saindane NS, Pagar KP, Vavia PR. Nanosuspension based in situ gelling nasal spray of carvedilol: development, in vitro and in vivo characterization. AAPS PharmSciTech. 2013;14:189-99. 